Operator Splitting and Approximate Factorization for Taxis-Diffusion-Reaction Models

A. Gerisch, J.G. Verwer

Modelling, Analysis and Simulation (MAS)

MAS-R0026 September 30, 2000 
Report MAS-R0026

ISSN 1386-3703

CWI

P.O. Box 94079

1090 GB Amsterdam

The Netherlands

$\mathrm{CWI}$ is the National Research Institute for Mathematics and Computer Science. CWI is part of the Stichting Mathematisch Centrum (SMC), the Dutch foundation for promotion of mathematics and computer science and their applications.

SMC is sponsored by the Netherlands Organization for Scientific Research (NWO). CWI is a member of ERCIM, the European Research Consortium for Informatics and Mathematics.

Copyright (C) Stichting Mathematisch Centrum P.O. Box 94079, 1090 GB Amsterdam (NL) Kruislaan 413, 1098 SJ Amsterdam (NL) Telephone +3120 5929333 Telefax +31205924199 


\title{
Operator Splitting and Approximate Factorization for Taxis-Diffusion-Reaction Models
}

\author{
A. Gerisch \\ Institut für Numerische Mathematik, Fachbereich Mathematik und Informatik, \\ Martin-Luther-Universität Halle-Wittenberg, Postfach, 06099 Halle (Saale), Germany \\ J.G. Verwer \\ $C W I$ \\ P.O. Box 94079, 1090 GB Amsterdam, The Netherlands \\ gerisch@mathematik.uni-halle.de, jan.verwer@cwi.nl
}

\begin{abstract}
In this paper we consider the numerical solution of 2D systems of certain types of taxisdiffusion-reaction equations from mathematical biology. By spatial discretization these PDE systems are approximated by systems of positive, nonlinear ODEs (Method of Lines). The aim of this paper is to examine the numerical integration of these ODE systems for low to moderate accuracy by means of splitting techniques. An important consideration is maintenance of positivity. We apply operator splitting and approximate matrix factorization using low order explicit Runge-Kutta methods and linearly implicit Runge-Kutta-Rosenbrock methods. As a reference method the general purpose solver VODPK is applied.
\end{abstract}

2000 Mathematics Subject Classification: Primary: 65M20. Secondary: 92B05, 92C99.

1998 ACM Computing Classification System: G.1.7 and G.1.8.

Keywords and Phrases: PDEs, Taxis-Diffusion-Reaction Problems, Method of Lines, Positivity, Rosenbrock methods, Operator Splitting, Approximate Matrix Factorization.

Note: Work carried out under subtheme MAS1.3 - Applications from the Life Sciences.

\section{Introduction}

\subsection{Taxis-diffusion-reaction equations}

The computation of solutions of partial differential equation (PDE) models from mathematical biology is of ever increasing importance for the understanding of biological processes, for the verification of hypothesis about the underlying biology and also for the application of such models to patient specific data in medicine. The complexity of the model equations nearly always necessitates the application of efficient numerical methods. Numerical solutions should be obtained within a reasonable short computation time to enable extensive parameter studies. Because the model parameters and the initial data are often known as crude approximations only, one is mostly satisfied with low to moderately accurate approximations. Despite the modest accuracy requirements, it remains important to resolve certain qualitative solution properties correctly, which sometimes requires a stricter accuracy. One such property is non-negativity, henceforth called positivity, of concentration values featuring in bio-chemical reactions. Violating positivity is highly undesirable because it may turn stable reactions into unstable ones which in turn may lead to numerical instabilities. Taking these considerations into account, in this paper we study the numerical solution of 
the taxis-diffusion-reaction system

$$
\begin{aligned}
\frac{\partial n}{\partial t} & =\varepsilon \Delta n-\nabla \cdot\left(n \sum_{i=1}^{l} f_{i}(\underline{c}) \nabla c_{i}\right)+f_{0}(n, \underline{c}), \\
\frac{\partial \underline{c}}{\partial t} & =D \Delta \underline{c}+\underline{g}(n, \underline{c})
\end{aligned}
$$

where the time and space dependent functions $n(t, \underline{x})$ and $\underline{c}(t, \underline{x})$ denote the density of a population and a vector of $l$ concentrations or densities of certain chemicals, respectively.

We consider this system on the unit square in space, $\underline{x} \in[0,1]^{2}$, and for finite time intervals, $t \in[0, T]$. The population might diffuse with diffusion constant $\varepsilon>0$ or exhibits no diffusion $(\varepsilon=0)$. A characteristic property is that the evolution of $n$ depends on gradients $\nabla c_{i}$ of the chemical concentrations - a process known as (chemo)taxis which adds advection terms to the population equation. The strength and the sign of the tactic influence of each chemical $c_{i}$ on $n$ is described by $f_{i}(\underline{c}), i=1,2, \ldots, l$. We focus on biological systems where the diffusion coefficient $\varepsilon$ is much smaller than the speed of migration induced by the taxis term or where there is no diffusion in the population at all. The reaction term $f_{0}(n, \underline{c})$ accounts for creation or loss of entities in the population. The chemical concentrations in $\underline{c}$ can also change by diffusion $(D$ is a diagonal matrix with non-negative entries), or be spatially bound (then the corresponding diagonal entry in $D$ is zero). Finally, reactions between the concentrations and the population density are modelled through the vector-valued function $\underline{g}(n, \underline{c})$. For our numerical investigations we consider two specific models, a tumour invasion model [1] and a tumour angiogenesis model [5].

\subsubsection{A tumour invasion model}

This model has three components, $n$ is the tumour cell density and $c_{1}$ and $c_{2}$ are the density of the extracellular matrix (ECM) and the concentration of the matrix degradative enzymes (MDE), respectively. The system reads

$$
\begin{aligned}
\frac{\partial n}{\partial t} & =\varepsilon \Delta n-\nabla \cdot\left(n \gamma \nabla c_{1}\right), \\
\frac{\partial c_{1}}{\partial t} & =-\eta c_{2} c_{1}, \\
\frac{\partial c_{2}}{\partial t} & =d_{2} \Delta c_{2}+\alpha n-\beta c_{2},
\end{aligned}
$$

and is provided with Neumann boundary conditions for $n$ and $c_{2}$,

$$
\underline{\zeta} \cdot(\nabla n)=0 \text { and } \underline{\zeta} \cdot\left(\nabla c_{2}\right)=0 \quad(\underline{\zeta}=\text { outward unit normal vector }) .
$$

We choose parameters as in [1],

$$
\varepsilon=0.001, \gamma=0.005, \eta=10, d_{2}=0.001, \alpha=0.1, \beta=0,
$$

and in addition use these parameters with zero diffusion for $n(\varepsilon=0)$. In this case we also apply Neumann boundary conditions for $n$. We emphasize that boundary conditions have no notable influence on the solution in our simulations because the cell density near the boundary is virtually zero during simulation time. The initial conditions for tumour cells and MDE (which are produced by the tumour) are concentrated in the centre of the domain and given by

$$
n(0, \underline{x})=c_{2}(0, \underline{x}):=\exp \left(-\frac{\|\underline{x}-(0.5,0.5)\|_{2}^{2}}{0.0025}\right) .
$$

A hypothetical heterogeneous ECM initial distribution is assumed as depicted in Figure 1 (left) [1]. The final simulation time for the described setup is $T=15$. Questions on this model are, for instance, how far tumour cells can invade into the surrounding tissue and under which circumstances the initial cell mass does break up into pieces. 

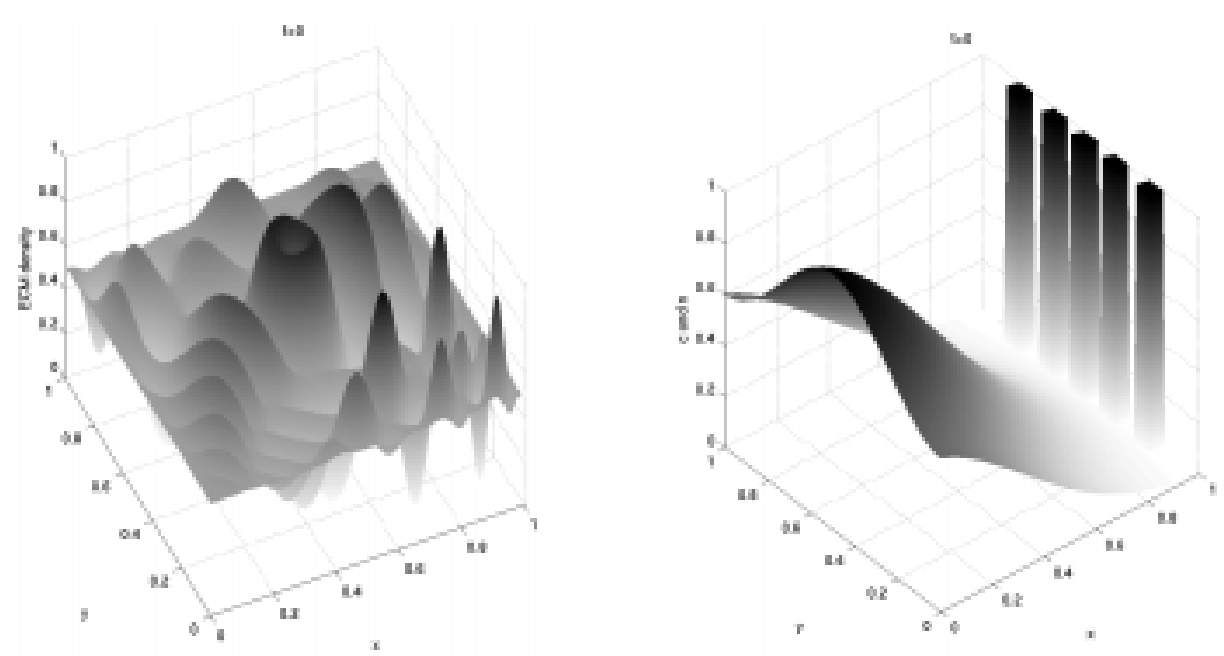

Figure 1: Heterogeneous ECM $\left(c_{1}\right)$ initial condition of Example 1.1.1 (left). Initial conditions for Example 1.1.2 (right) - the smooth function is the initial TAF concentration $c_{2}$ and the function which is zero except for the blocks on the right boundary is the initial cell distribution $n$.

\subsubsection{A tumour angiogenesis model}

Angiogenesis is the outgrowth of new blood vessels from a pre-existing vascular network. This model considers the case that this process is induced by a tumour which aims to establish a connection to the blood network - and hence nutrient supply - in order to be able to grow further. It has two components. Component $n$ is the density of endothelial cells which line the blood vessels and hence $n$ is a measure of the density of the developing network. The concentration of tumour angiogenesis factor (TAF) is denoted by $c_{1}$. TAF is secreted by the tumour and stimulates blood vessel growth. The model equations are given by [5]

$$
\begin{aligned}
\frac{\partial n}{\partial t} & =\varepsilon \Delta n-\nabla \cdot\left(n \kappa \nabla c_{1}\right)+\mu n(1-n) \max \left\{0, c_{1}-c^{\star}\right\}-\beta n, \\
\frac{\partial c_{1}}{\partial t} & =\Delta c_{1}-\lambda c_{1}-\frac{\alpha n c_{1}}{\gamma+c_{1}} .
\end{aligned}
$$

We consider two cases, the case with diffusion for $n$ with $\varepsilon=0.001$ and the case without diffusion as is also done in $[7,8]$. Otherwise we take the parameter values from [5] given by

$$
\alpha=10, \beta=4, \gamma=1, \kappa=0.7, \lambda=1, \mu=100, c^{\star}=0.2 .
$$

A single tumour is located on the left edge of the spatial domain $(x=0)$ and we choose the initial condition for the TAF concentration $c_{1}$ as given in Figure 1 (right). This figure (right) also depicts the initial cell concentration $n$ corresponding to a parent blood vessel on the right boundary of the domain $(x=1)$ with some already developed capillary sprouts. We prescribe the following boundary conditions for $c_{1}(t, x, y)$,

$$
c_{1}(t, 0, y)=c_{1}(0,0, y), \quad c_{1}(t, 1, y)=c_{1}(0,1, y), \quad c_{1, y}(t, x, 0)=c_{1, y}(t, x, 1)=0 .
$$

An inflow boundary condition for $n$ on the right boundary only is used if $\varepsilon=0$,

$$
n(t, 1, y)=n(0,1, y) \text {. }
$$

In the other case, $\varepsilon=0.001$, we add Neumann boundary conditions for $n$ on the remaining part of the boundary.

The final simulation time for the described setups are $T=1.3$ for $\varepsilon=0$ and $T=1.1$ for $\varepsilon=0.001$. Thereafter the assumptions underlying the model do not hold anymore because the blood vessels have reached the tumour and other processes take over. 


\subsection{The numerical approach}

We obtain numerical solutions of these problems by following the 'method of lines'. That means that we consider the discretization of the spatial operators and the time integration separately. For the spatial discretization we use the Eulerian grid approach on an equidistant grid. The diffusion terms are approximated by second order central differences. For our application this standard diffusion discretization works satisfactorily. For the taxis term standard central differencing cannot be used as this would lead to wiggles and negative concentrations in the solution. Similar as with the more common advection dominated advection-diffusion problems, these arise in the vicinity of steep gradients when the transport of $n$ induced by the taxis term is much stronger than the diffusion transport. We therefore approximate the taxis term by the flux limited, upwind biased $\kappa=\frac{1}{3}$ discretization with the van Leer flux limiter (second order), see $[7,13]$. For the current taxis problem this special discretization has been extensively examined in [7]. We refer to that paper for details and for the discretization of the boundary conditions.

The result of the spatial discretization is an initial-value problem for a huge system of stiff, nonlinear ordinary differential equations (ODEs) which remains to be numerically integrated in time. We denote this system by

$$
y^{\prime}(t)=F(t, y(t)), \quad y(0)=y_{0}, \quad t \in[0, T] .
$$

Hence the solution vector $y$ is the grid function containing all semi-discrete approximations to the population density $n$ and the chemicals $c_{i}$. We are especially interested in the numerical integration of these large ODE systems by means of splitting techniques. Using low order explicit Runge-Kutta methods and linearly implicit Runge-Kutta-Rosenbrock methods, we compare two such techniques, operator splitting (OPS) and approximate matrix factorization (AMF).

The usefulness of splitting techniques becomes evident when we write the vector function $F$ as

$$
F(t, y)=F_{0}(t, y)+F_{1}(t, y),
$$

where we have collected all terms from the taxis discretization in $F_{0}$ and all diffusion and reaction terms in $F_{1}$. We separate these terms because the system $y^{\prime}(t)=F_{1}(t, y(t))$ generally requires an implicit treatment because of stiffness, whereas the semi-discrete taxis system $y^{\prime}(t)=F_{0}(t, y(t))$ is better solved explicitely because this is often more efficient. The splitting techniques OPS and AMF make use of this separation and treat $F_{0}$ and $F_{1}$ differently. We can further split $F_{1}$ by separating terms of $x$ - and $y$-diffusion discretization and reaction terms,

$$
F_{1}(t, y)=F_{D_{x}}(t, y)+F_{D_{y}}(t, y)+F_{R}(t, y) .
$$

This secondary splitting will be used to reduce linear algebra costs.

\subsection{Description of contents}

In Section 2 we review some positivity results for explicit Runge-Kutta (ERK) methods and give two methods (modified Euler (ME) and RK32 [9]) which are appropriate for the solution of our semi-discrete taxis system $y^{\prime}(t)=F_{0}(t, y(t))$. Positivity of the numerical solution is important in our application because the population density $n$ feeds back into the reaction terms and negative values lead to unstable behaviour (negative values make also no sense biologically). The population density contains steep fronts in the solution and this causes problems if the time integration method is not selected carefully.

In Section 3 we propose to apply the linearly implicit 2-stage, 2nd order Rosenbrock-W method ROS2 earlier used in [23]. This is done by using an inexact Jacobian which does not take the Jacobian matrix of $F_{0}$ into account. The resulting method therefore only requires the solution of linear systems coming from the Jacobian matrix of the diffusion-reaction function $F_{1}$. These matrices 
are broadly banded. To make the required numerical linear algebra amenable, the approximate matrix factorization (AMF) technique is applied. The resulting scheme is named AMF-ME because the underlying ERK method is ME. However, the ERK scheme RK32 has more favourable stability and positivity properties than ME. Therefore we also propose an $L$-stable, 3 -stage, 2 ndorder Rosenbrock-W method with underlying explicit scheme RK32. Our hope is that the good properties of RK32 and of Rosenbrock-W methods combine in the resulting scheme which we refer to as AMF-RK32.

In Section 4 we turn our attention to operator splitting (OPS). While AMF can be viewed as splitting on the level of the linear algebra, OPS involves splitting at the ODE level itself. The OPS technique is well-known and has been proven useful in a wide variety of multi-dimensional time-dependent PDE calculations. For example, the application of OPS to a model describing a spatial pattern formation process of bacteria is discussed by Tyson et al. in [22]. In most cases OPS introduces a splitting error (see e.g. [17]), but it has the great advantage that different parts of the ODE are separated more strictly and special purpose solvers can be applied. In this section we present two Strang-type operator splitting methods which treat ODEs with right-hand side $F_{0}$ explicitly (ME or RK32) and ODEs with right-hand side $F_{1}$ with ROS2 employing AMF. The resulting schemes are named OPS-ME and OPS-RK32.

Altogether we have four different methods from two different approaches. These are compared and evaluated in Section 5 for application to the invasion and angiogenesis models. Finally, we summarize our results and present conclusions in Section 6.

\section{Positivity of ERK methods}

In this section we consider initial-value problems for systems of ODEs

$$
y^{\prime}(t)=f(t, y(t)), \quad t \geq t_{0}, \quad y\left(t_{0}\right)=y_{0} .
$$

We assume that $f$ has the property

$$
f \text { is continuous and (8) has a unique solution for all } t_{0} \in \mathbb{R} \text { and all } y_{0} \in \mathbb{R}^{m} \text {. }
$$

The IVP (8) is called positive if $f$ has the property (9) and solution $y(t) \geq 0$ for all $t \geq t_{0}$ whenever $y_{0} \geq 0$. The following lemma from [11] characterizes positive ODE systems (see also [13]).

\section{Lemma 1}

Let $f$ satisfy condition (9). The IVP (8) associated with this function is positive if and only if for all $t$ and any vector $v \in \mathbb{R}^{m}$ and all $i=1(1) m$,

$$
v_{i}=0, v_{j} \geq 0 \text { for all } j \neq i \quad \Rightarrow \quad f_{i}(t, v) \geq 0 .
$$

The proposed discretization in space of our models leads to ODE systems (5) which satisfy the conditions of the lemma (this is also true if $F_{0}$ or $F_{1}$ are zero) [7, 13]. Hence it is natural to seek numerical approximations which also remain positive if time proceeds. Difficulties arise mainly from the discretization of the taxis part and these occur if the cell density equation has steep moving front solutions. These fronts typically occur when the diffusion part is much smaller than the taxis part, which is the case for our models. As stated in the introduction, we want to solve ODEs with right-hand side taxis function $F_{0}$ with low order, positive explicit methods. In [9] this issue is considered for explicit Runge-Kutta (ERK) methods. Here we repeat the main results from that paper.

Let $g(t) \geq 0$ be a given continuous, vector-valued function, $\alpha \geq 0$ a real number and define

$$
\mathcal{L}_{g}^{+}(\alpha):=\left\{f \mid f(t, y)=P y+g(t) \text { where } P \in \mathbb{R}^{m, m}, P+\alpha I \geq 0\right\} .
$$


IVPs with right-hand side functions taken from this class are positive. Positivity of one-step methods applied to problems $(8)$ from the class $\mathcal{L}_{g}^{+}(\alpha)$ has been investigated in [3]. There it is proven that explicit methods applied with time step size $\tau$ yield positive numerical solutions if $\alpha \tau \leq \mu$ (in the case of $g=0$ ), where $\mu$ is the so-called threshold factor of absolute monotonicity of the stability polynomial of the method. These threshold factors are considered in [15], where it is shown that $\mu=1$ for all $s$-stage ERK methods of order $s$. However, at the cost of one additional function evaluation, one can achieve $\mu=2$ ( $s$-stage ERK methods of order $s-1$ [15]), which means a doubling of the maximum value of $\tau$. For 3-stage methods of order 2 this is the case if the stability polynomial takes the form $1+z+\frac{1}{2} z^{2}+\frac{1}{12} z^{3}$. The Butcher array of the corresponding class of ERK methods is given in Figure 2 (left). We note that this stability polynomial has also an enlarged stability region compared to $s$-stage ERK methods of order $s$ with $s=2,3$.

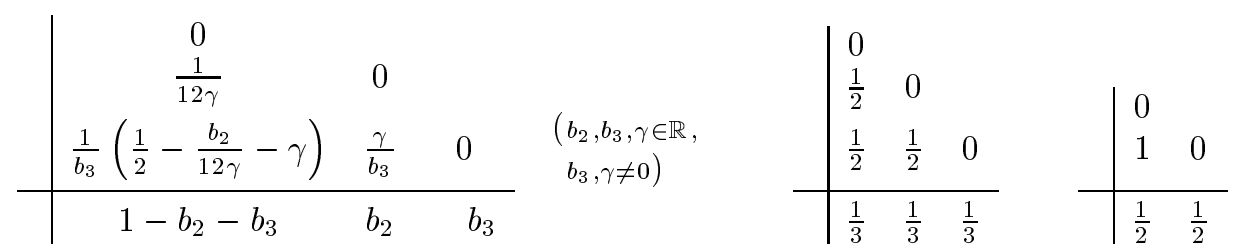

Figure 2: Butcher arrays for a general 3-stage, 2nd-order ERK method with optimal positivity on the class $\mathcal{L}_{0}^{+}(\alpha)$ (left), for method RK32 (middle) and method ME (right).

The free parameters in Figure 2 (left) can be fixed by considering positivity for nonlinear problems. As far as we know two approaches exist. One is by Horváth [11]. He considers subclasses of dissipative, positive problems. Here the characteristic constant of the ERK scheme is the radius of absolute monotonicity of the method (see also [16]). This radius is bounded from above by the threshold factor of absolute monotonicity. The other approach was proposed by Shu and Osher [20], see also [13]. Here the ERK scheme is written as a convex combination of forward Euler steps for which the time step restriction for positivity for specific problems is easily established. This results in a characteristic constant called positivity factor in terms of the parameters of the convex combination. The maximum allowable time step size for positivity of the method is then proportional to this positivity factor.

It turns out [9] that there exists exactly one choice of parameters in Figure 2 (left) such that the resulting method has a radius of absolute monotonicity equal to two and this is optimal. This method has $b_{2}=b_{3}=1 / 3$ and $\gamma=1 / 2$, see Figure 2 (middle) and is called RK32. RK32 is also the only method with a non-zero radius of absolute monotonicity from this class which has a positivity factor two and this is also optimal. Further, the scheme RK32 is also positive when applied to problems from $\mathcal{L}_{g}^{+}(\alpha)$ under the step size restriction $\alpha \tau \leq \mu=2$ and again this is optimal within the method class of Figure 2 (left).

Within the OPS and AMF approach we will compare RK32 with the popular 2-stage, 2nd-order modified Euler scheme (ME), see Figure 2 (right). ME has threshold factor, radius of absolute monotonicity and positivity factor equal to one and is hence optimal in the class of 2-stage, 2ndorder ERK methods.

\section{The Rosenbrock AMF methods}

\subsection{AMF-ME}

Verwer et al. [23] successfully applied the 2-stage, 2nd-order Rosenbrock-W method ROS2 to advection-diffusion-reaction problems from atmospheric air pollution modelling. The methodology applied in that paper also appears to be of interest for taxis-diffusion-reaction equations. Because 
our current models give rise to autonomous systems, we consider for ROS2 the autonomous form as used in [23]. This form, with the matrix $A$ appearing at the left side only, reads

$$
\begin{aligned}
y_{n+1} & =y_{n}+\tau\left(\frac{3}{2} k_{1}+\frac{1}{2} k_{2}\right), \\
y_{n+1}^{(1)} & =y_{n}+\tau k_{1}, \\
(I-\tau \gamma A) k_{1} & =F\left(y_{n}\right) \\
(I-\tau \gamma A) k_{2} & =F\left(y_{n+1}^{(1)}\right)-2 k_{1},
\end{aligned}
$$

where $y_{n}$ is the approximation to $y\left(t_{n}\right), \tau$ is the step size taken from $t_{n}$ to $t_{n+1}$ and $A$ is an arbitrary approximation to the Jacobian matrix $F^{\prime}\left(y_{n}\right)$ (W-method property). If we take for $A$ the zero matrix, the modified Euler method ME is obtained. We have 2nd-order consistency for any matrix $A$ and any value of the parameter $\gamma$. L-stability is obtained for $\gamma=1 \pm \sqrt{2} / 2$. We select the smaller value $\gamma=1-\sqrt{2} / 2$ because with the larger one there does not exist a $\tau>0$ such that the method is positive on class (10) (this is the case for all $\gamma>1 / 2$ ). The auxiliary value

$$
y_{n+1}^{(1)}=y_{n}+\tau k_{1}
$$

provides a 1st-order embedded solution which will be used for variable step size control.

Low order Rosenbrock methods are efficient for a wide range of stiff ODE problems, see e.g. [10]. However, applying ROS2 involves two linear solves with the matrix $I-\tau \gamma A$. Consequently, choosing $A$ equal to the full Jacobian

$$
F^{\prime}\left(y_{n}\right)=F_{0}^{\prime}\left(y_{n}\right)+F_{1}^{\prime}\left(y_{n}\right)=F_{0}^{\prime}\left(y_{n}\right)+F_{D_{x}}^{\prime}\left(y_{n}\right)+F_{D_{y}}^{\prime}\left(y_{n}\right)+F_{R}^{\prime}\left(y_{n}\right)
$$

seems not practical due to the huge matrix size (semi-discrete PDE problems). Moreover, due to the flux-limiter used in the taxis discretization, $F$ is only Lipschitz continuous so that the Jacobian might not even exist. The same situation occurs in the air pollution application of [23] where ROS2 is applied with a matrix $A$ approximating the true Jacobian. For the taxis models we use a similar approximation which yields the following choice for the matrix $I-\tau \gamma A$,

$$
I-\tau \gamma A:=\left(I-\tau \gamma F_{R}^{\prime}\left(y_{n}\right)\right)\left(I-\tau \gamma F_{D_{y}}^{\prime}\left(y_{n}\right)\right)\left(I-\tau \gamma F_{D_{x}}^{\prime}\left(y_{n}\right)\right) .
$$

This approximation is made in two steps. First, we have neglected the taxis Jacobian $F_{0}^{\prime}\left(y_{n}\right)$ which overcomes the possible difficulty of non-existence. This choice further underlies the assumption that when applied to

$$
y^{\prime}(t)=F_{0}(y(t))
$$

the explicit method ME has satisfactory positivity and stability properties. Second, we have approximated the remainder matrix $I-\tau \gamma F_{1}^{\prime}\left(y_{n}\right)$ by the factorized expression (12). With this factorization we avoid linear solves which are still expensive because $F_{1}^{\prime}\left(y_{n}\right)$ has a bandwidth $\mathcal{O}\left(h^{-1}\right), h$ denoting the spatial grid size. For efficiency it is important that the matrices involved are banded with a small bandwidth independent of $h$. This holds with (12). This property is especially profitable for the fine spatial resolutions required in our models to resolve steep fronts for the cell density. The factorization is known as 'Approximate Matrix Factorization' (AMF) which is in use for a long time already for solving multi-space dimensional time-dependent PDE problems, see e.g. [2, 6, 12, 14, 18].

AMF does not affect the order of consistency because ROS2 is of 2nd-order for any choice of $A$. It does of course affect the stability of the original ROS2 used with $A=F^{\prime}\left(y_{n}\right)$. In [23] it is argued that with (12) the stability of the resulting AMF-ME method is mainly governed by the stability of method ME applied to the advection part only. A similar conclusion can be drawn for the taxis-diffusion-reaction problems. If the split matrices do not commute then the order of the factors in the AMF can be important for the performance of the method and the best choice is problem specific. 


\subsection{AMF-RK32}

In Section 2 we have argued that method RK32 from Figure 2 will solve the semi-discrete taxis system (13) with less time steps than method ME. We have therefore searched for a 3-stage Rosenbrock-W method of 2nd-order with underlying ERK scheme RK32. After some standard calculations we have chosen the following formula,

$$
\begin{aligned}
(I-\tau \gamma A) k_{i} & =F\left(y_{n}+\frac{\tau}{2} \sum_{j=1}^{i-1} k_{j}\right)+\tau A \sum_{j=1}^{i-1} \gamma_{i j} k_{j}, \quad i=1,2,3 \\
y_{n+1} & =y_{n}+\frac{\tau}{3} \sum_{i=1}^{3} k_{i}
\end{aligned}
$$

where

$$
\begin{gathered}
\gamma_{21}=-\left(3 \gamma+\gamma_{31}+\gamma_{32}\right), \quad \gamma_{32}=1 / 2-3 \gamma \\
\gamma_{31}=\frac{-1}{1+2 \gamma_{32}}\left(6 \gamma^{3}-12 \gamma^{2}+6\left(1+\gamma_{32}\right) \gamma+2 \gamma_{32}^{2}-\frac{1}{2}\right) \\
\gamma=1-\frac{1}{2} \sqrt{2} \cos \theta+\frac{1}{2} \sqrt{6} \sin \theta
\end{gathered}
$$

with $\theta=\frac{1}{3} \arctan \left(\frac{1}{4} \sqrt{2}\right)$. The parameters were chosen such that the method is L-stable and 3rd-order for constant coefficient linear problems. The value for $\gamma$ is approximately 0.43586652 . The intermediate approximation

$$
y_{n+1}^{(2)}=y_{n}+\frac{\tau}{2}\left(k_{1}+k_{2}\right)
$$

provides a cheap 1st-order embedded solution for variable step size control. The scheme is to be rearranged such that $A$ appears only on the left-hand side of the equations, similar as for ROS2. One then can apply the method with the approximate matrix factorization (12) in the same manner. We call the resulting method AMF-RK32. The factorization does not affect the order of accuracy because (14) is a Rosenbrock-W method. Stability can be analysed in the same way as in [23].

\section{The operator splitting methods}

Whereas the AMF methods perform a splitting at the linear algebra level, it is also possible to directly split at the problem level, that is, to apply operator splitting. Like approximate factorization, operator splitting is a popular approach for solving multi-space dimensional time-dependent PDE problems. Operator splitting has been considered in [7] for the tumour angiogenesis model. The method proceeds as follows. Given an approximation $y_{n}$ at time $t_{n}$ and a step size $\tau$, we compute

$$
y_{n+1}=\Phi_{0}\left(\frac{\tau}{2}, t_{n}+\frac{\tau}{2}\right) \Phi_{1}\left(\tau, t_{n}\right) \Phi_{0}\left(\frac{\tau}{2}, t_{n}\right) y_{n}
$$

where $\Phi_{0}$ and $\Phi_{1}$ are approximate evolution operators of $F_{0}$ and $F_{1}$, respectively. Specifically, $\Phi_{i}(\tau, \tilde{t}) u$ approximates the solution of the initial-value problem (here in non-autonomous form)

$$
y^{\prime}(t)=F_{i}(t, y(t)), \quad t \geq \tilde{t}, \quad y(\tilde{t})=u
$$

at $t=\tilde{t}+\tau$. This form is known as Strang-splitting [21]. If the operators $\Phi_{i}$ are at least 2ndorder accurate approximations of the exact evolution operators, then the order of consistency of the 
approximation (15) equals two. The stability and positivity of (15) is determined by the associated properties of $\Phi_{0}$ and $\Phi_{1}$.

It is effective to select an explicit method for $\Phi_{0}$ and an implicit method for $\Phi_{1}$. We have already all necessary ingredients available and will use the ROS2 scheme with AMF for the implicit method, see Section 3.1, and replace $F$ by $F_{1}$ in the method (11). The explicit method will be either ME or RK32. We refer to the resulting operator splitting schemes as OPS-ME and OPS-RK32, respectively. Operator splitting is applied in the order given in (15) because then we use only half the step size of the splitting step for the explicit method. This doubles the stability and positivity domain of the explicit method and hence is expected to lead overall to less time steps.

\section{$5 \quad$ Numerical Experiments}

Following standard practice, we have implemented the four methods with variable step sizes [10, 19$]$. The embedded first order solution of the Rosenbrock scheme is used to obtain an estimate of the local error of the current step in the two AMF methods. The time step is selected on the basis of an error per step (EPS) control which aims to keep this estimate below a mixed (relative and absolute) threshold depending on the user supplied tolerance TOL ( $=$ ATOL $=$ RTOL). The second order solution is used to advance an accepted step (local extrapolation [19, p. 342]). The two OPS methods use Richardson extrapolation to obtain a local error estimate of the current step and then the same EPS control to select the step size. They step forward with the solution obtained after two half-steps (doubling [19, p. 364], no local extrapolation to third order).

Jacobians are evaluated at the beginning of a time step (AMF) or at the beginning of a Richardson step (OPS). We compute finite difference approximations to the true Jacobians of the split functions.

We compare the solutions, $y_{\text {comp }}$, of the ODE systems at final time (corresponding to the examples considered here) computed with our methods against reference solutions $y_{r e f}$. We obtain these reference solutions of the ODE systems with the standard integrator VODPK [4] requiring a tolerance $T O L=10^{-12}$. Hence spatial errors play no role in the error measurements but splitting errors do. The error estimate $\operatorname{err}:=\left\|y_{\text {comp }}-y_{\text {ref }}\right\|$ between computed solutions and reference solutions is measured in the scaled $l_{2}$-norm,

$$
\|v\|=\left(\frac{1}{m} v^{\top} v\right)^{1 / 2}, \quad v \in \mathbb{R}^{m} .
$$

This norm is also used in the figures below.

In all test cases the four integration schemes are run for seven tolerance values $T O L=10^{-3}, 10^{-3.5}$, $\ldots, 10^{-6}$.

By way of comparison we also apply VODPK using the same range of tolerance values. VODPK is a variable-coefficient ODE solver with the preconditioned Krylov method GMRES for the solution of linear systems. It is based on the VODE and LSODPK packages. We use this method without preconditioning, with default parameters and set $\mathrm{MF}=21$ (method based on BDF formulas up to order 5 with a scaled, preconditioned, incomplete version of GMRES).

\subsection{Tumour Invasion Model - Example 1.1.1}

The solution $n$ of the cell density equation of this problem has an initial peak in the centre of the domain. This peak spreads outward moving up gradients of the ECM density $c_{1}$ which is heterogeneous initially. This leads to a heterogeneous pattern in the cell density solution. These patterns are sharper if there is no cell diffusion (a break up of the initially compact cell mass can be observed) and more smeared with cell diffusion (the break up of cell mass is not so pronounced in this case). The total cell mass in the domain is a conserved quantity of the model. The tumour cells release MDE $\left(c_{2}\right)$ which (slowly) diffuses within the spatial domain. MDE in turn degrades 
ECM and hence leads to new gradients in the ECM density which give rise to further migration of the cells. The most interesting solution of this model is the cell density and Figure 3 gives solution plots at three different output times for the cases with and without cell diffusion.
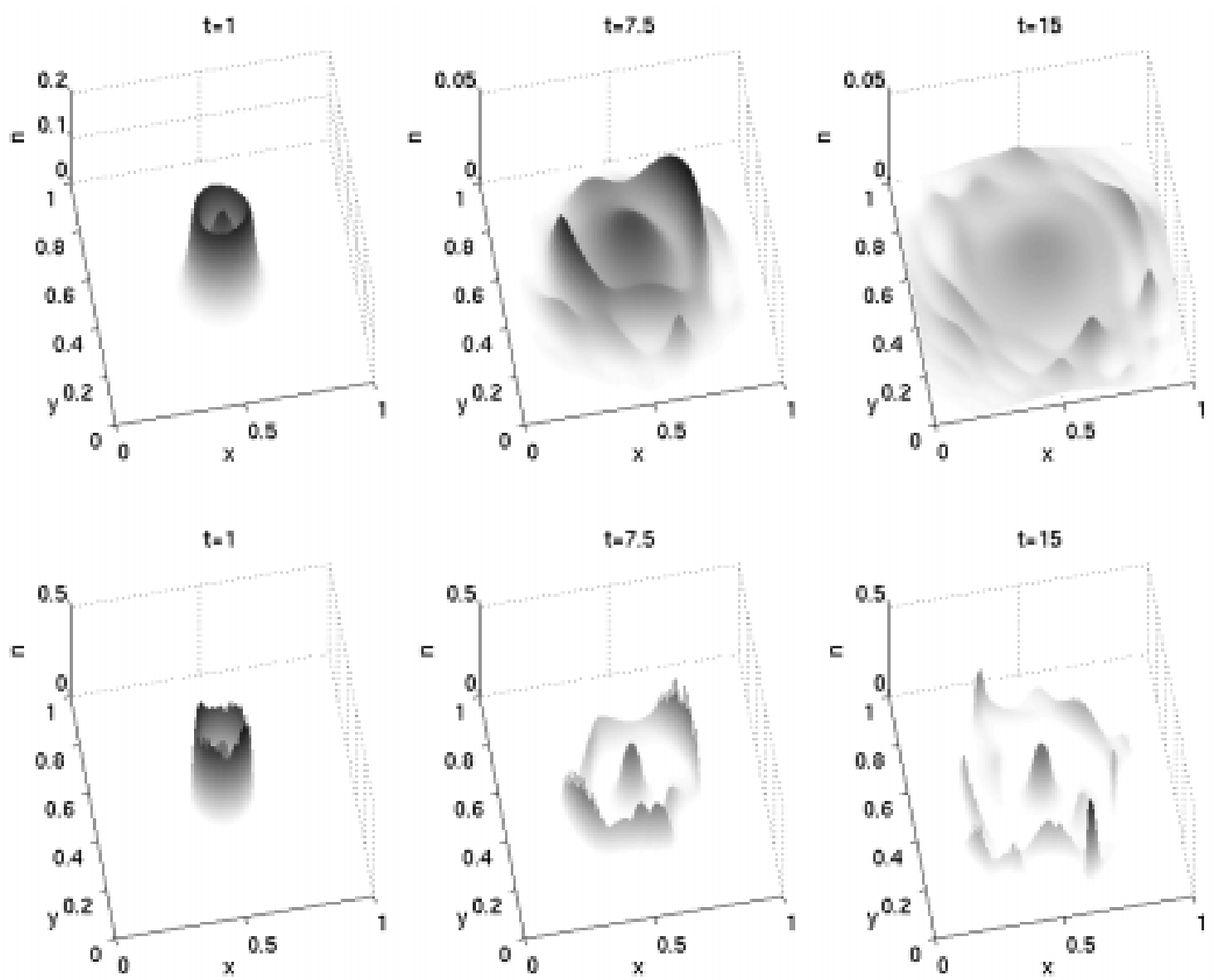

Figure 3: Simulation results for the cell density equation of the tumour invasion model. The top row is with cell diffusion and the bottom row without. The output times are given in the title of each plot.

Figures 4 and 5 give accuracy-CPU time plots for the tumour invasion model with and without cell diffusion, respectively. The left-hand plots correspond to a spatial grid width of $h=1 / 100$ and the right-hand plots to $h=1 / 200$.

VODPK turns out to be very efficient for this example. Due to increasing stiffness, this advantage decreases for the finer grid resolution and more significantly if the (small) diffusion coefficient $d_{2}=0.001$ is enlarged by a factor of 10 (see Figure 6) or 100 .

We note that the AMF schemes can be applied with even less stringent tolerance requirements ( e.g. up to $T O L=10^{-2}$ ) in the case with cell diffusion (Figure 4) and then these schemes outperform VODPK (for consistency we do not plot these data points here). In case of cell diffusion, and in general for the OPS schemes, slight stability problems were observed for less stringent values of TOL.

We further observe that a decrease of the required accuracy TOL by a factor 10 results in an achieved accuracy improvement of only a factor $\approx 5.5$ for both OPS schemes but of a factor $\approx 10$ for the AMF methods (the numbers are for the $h=1 / 100$ case with cell diffusion). This behaviour is caused by the different local error control mechanisms which are employed in the codes and is 

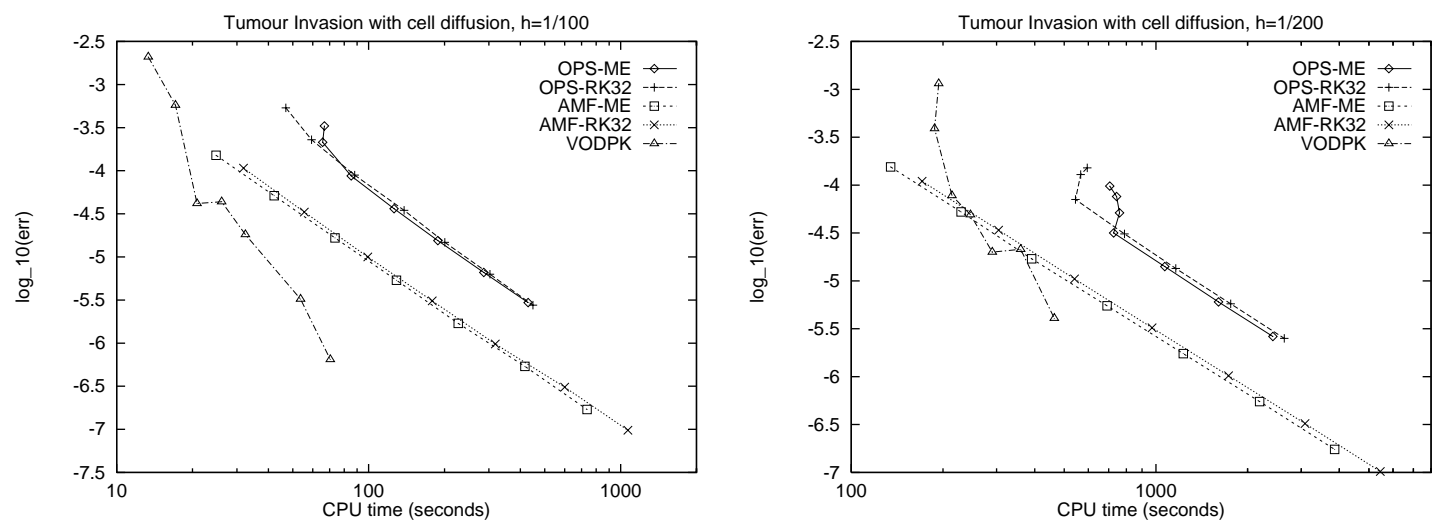

Figure 4: Results for the tumour invasion model with cell diffusion on a spatial grid with $h=1 / 100$ (left) and $h=1 / 200$ (right).
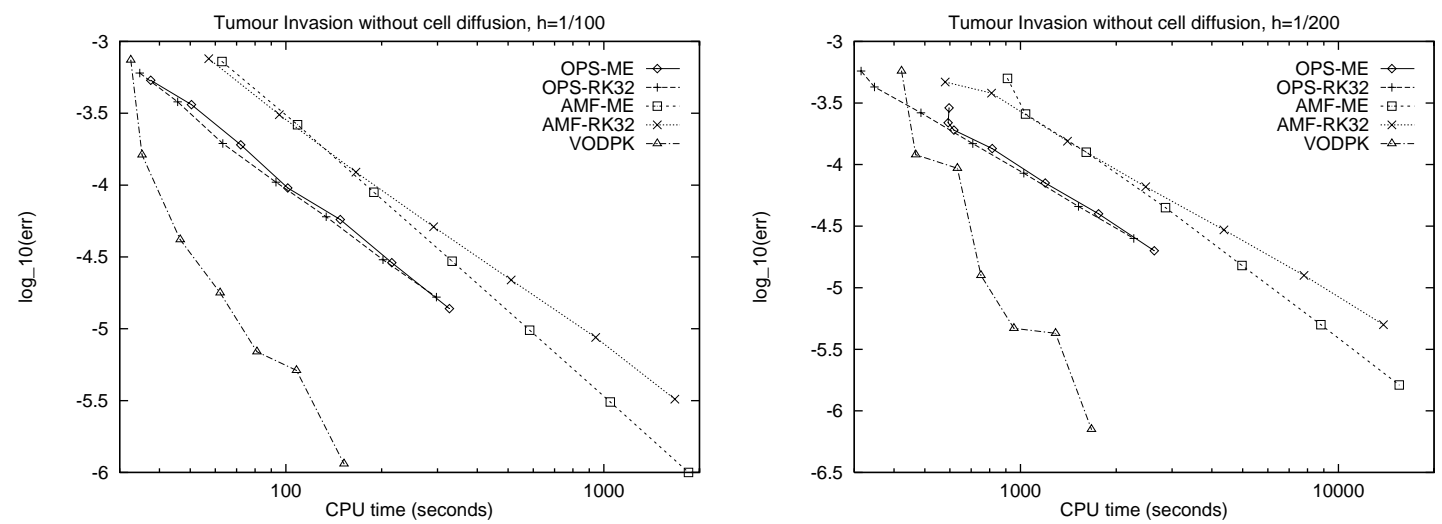

Figure 5: Results for the tumour invasion model without cell diffusion on a spatial grid with $h=1 / 100$ (left) and $h=1 / 200$ (right).

explained in detail in $\left[19\right.$, p. 350]. The true (global) error $e_{n}=y\left(t_{n}\right)-y_{n}$ satisfies approximately (under suitable assumptions)

$$
e_{n} \sim T O L
$$

for the error per step control with local extrapolation as used in the AMF codes but only

$$
e_{n} \sim T O L^{p /(p+1)}
$$

for the error per step control (without local extrapolation) as used in the OPS schemes $(p=2)$. The same proportionality factors multiplied by the step size $\tau$ result for the controlled local errors [19, p. 339 and p. 344]. These results suggest that we should expect a factor of $\approx 4.6$ for the OPS schemes which we clearly have. Also, we should be able to obtain tolerance proportionality for the OPS schemes if we require modified tolerances $T O L_{m o d}:=T O L^{3 / 2}$ and Figure 7 shows the expected results: we achieve a factor of $\approx 12$. (The same behaviour but with marginally smaller numbers is observed for the case $h=1 / 100$ without cell diffusion.)

We remark that the very stringent (modified) tolerance requirements lead to many rejected steps in the OPS methods in the case without cell diffusion. This is not the case for more relaxed tolerances and also not if cell diffusion is present in the model. The rejected steps are not caused by small negative solution values but rather by the less smooth solution in this case which makes 

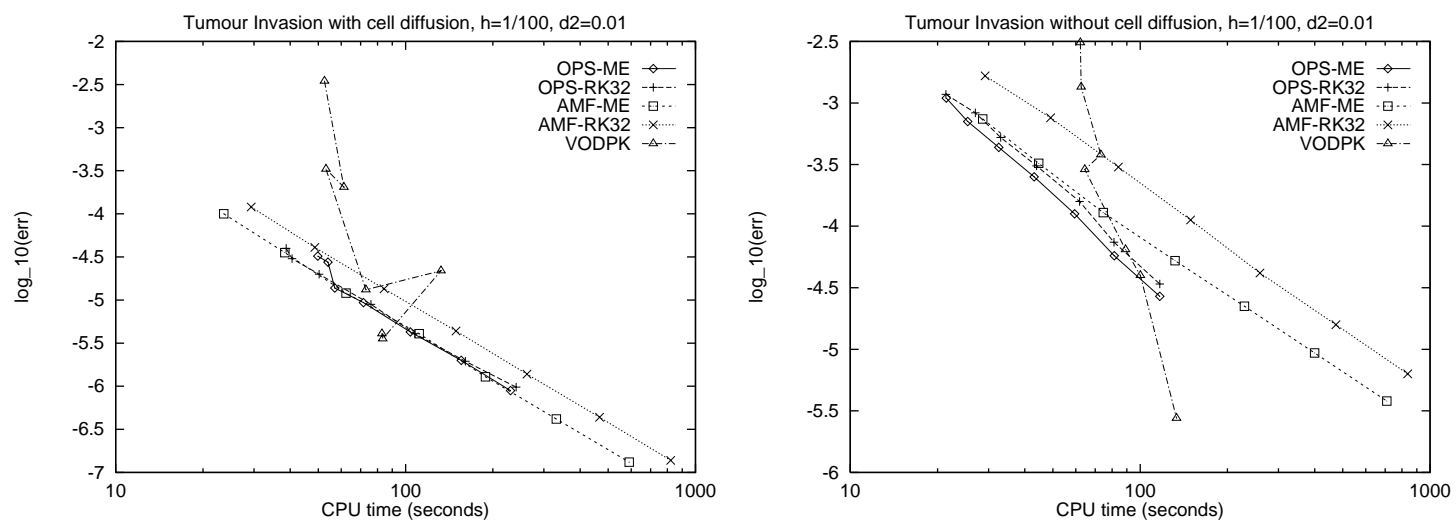

Figure 6: Results for the tumour invasion model with (left) and without (right) cell diffusion on a spatial grid with $h=1 / 100$. We changed the model parameter $d_{2}$ to a value of 0.01 in this experiment.

high accuracies difficult to attain. Except for this situation, there are only a few rejected steps for all codes and test cases.
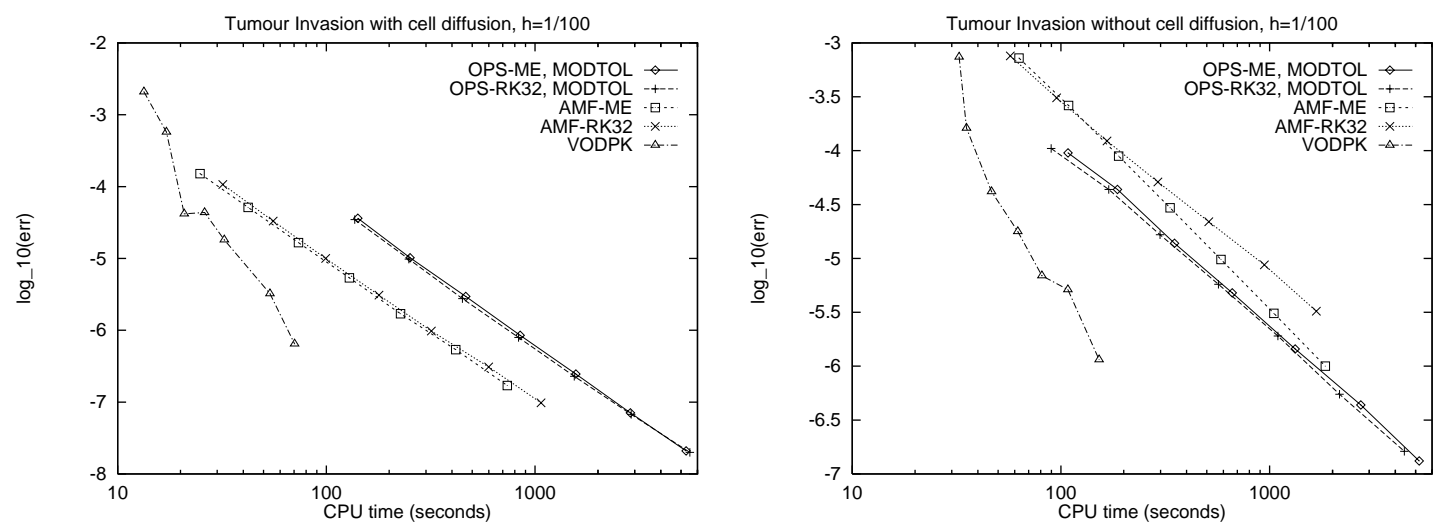

Figure 7: Results for the tumour invasion model with (left) and without (right) cell diffusion on a spatial grid with $h=1 / 100$. The OPS methods are applied with modified tolerances here.

Returning to Figures 4 and 5, we clearly see that the AMF schemes are more suitable than the OPS methods for the test case with cell diffusion; without cell diffusion, the situation is the opposite and the OPS schemes generally demonstrate a better performance.

It can also be observed that for cruder tolerances the methods based on RK32 have a slightly improved behaviour compared to the corresponding methods based on ME. We credit this advantage to the improved stability of RK32.

Finally, all tested codes preserve the initial cell mass up to a difference of the size of unit round off.

\subsection{Tumour Angiogenesis Model - Example 1.1.2}

The solution $n$ of the equation for the endothelial cell density of this problem has initially peaks near the right boundary of the domain. The cells there are migrating to the left - forming a stream which moves up the present TAF $\left(c_{1}\right)$ gradient as time proceeds. No cell proliferation takes place in the beginning of the simulation because the $c_{1}$ concentration at the cells is below the threshold $c^{\star}$. Later proliferation leads to a strong, local increase of the cell density. The cells also take up TAF. This results in changes in the TAF gradients and causes lateral cell movement and 
hence a widening of the cell streams. The cell streams turn towards the centre of the TAF source (the tumour) once they are close enough to the left boundary. Figure 8 gives cell density plots at three different output times for the model with and without cell diffusion. Note that the process proceeds faster if cell diffusion is present and that in this case also the lateral cell movement is more pronounced (leading to a closed wave front towards final time).
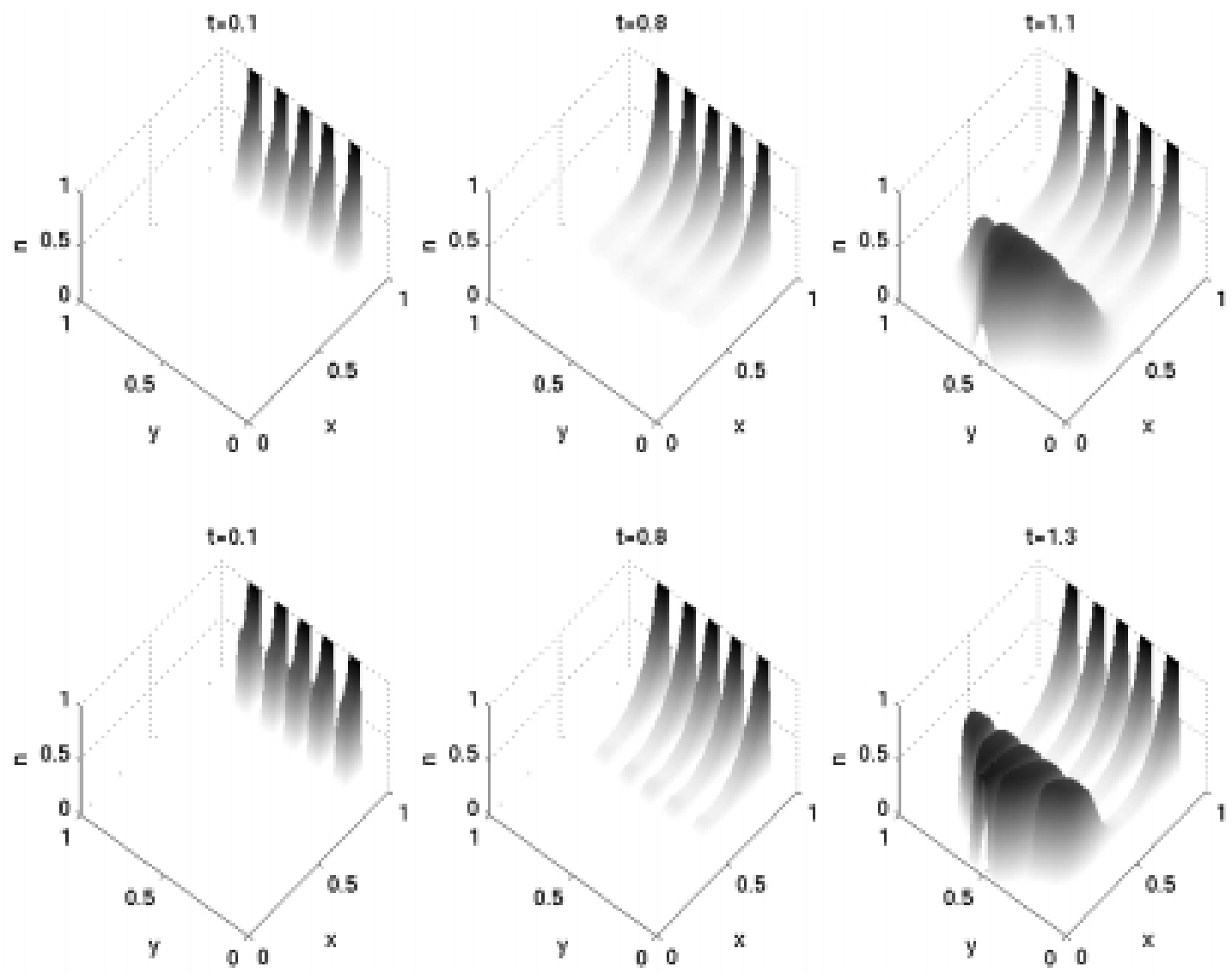

Figure 8: Simulation results for the cell density equation of the tumour angiogenesis model. The top row is with cell diffusion and the bottom row without. The output times are given in the title of each plot.

Figures 9 and 10 give accuracy-CPU time plots for the angiogenesis model with and without cell diffusion, respectively. The left-hand plots correspond to a spatial grid width of $h=1 / 100$ and the right-hand plots to $h=1 / 200$.

In all four test situations and up to moderate accuracy the AMF and OPS schemes are clearly much more efficient than the standard code VODPK. This is especially true for the finer grid resolution (right-hand plots) due to increasing stiffness. VODPK is more efficient for higher accuracy demands because of its higher order. However, we note that the point of intersection between the VODPK curve and the OPS-RK32 curve is at a higher achieved accuracy on the finer grid, i.e., if the spatial accuracy is increased then the splitting schemes are also more efficient for higher temporal accuracy.

Also we observe that the OPS schemes are more efficient than the AMF methods and this observation is independent of the choice of the cell diffusion coefficient $\varepsilon$. This is in contrast to the tumour invasion test case. In accordance with the tests from the previous section (but more evident), we see that the splitting methods based on RK32 are more suitable than those based on ME for the 

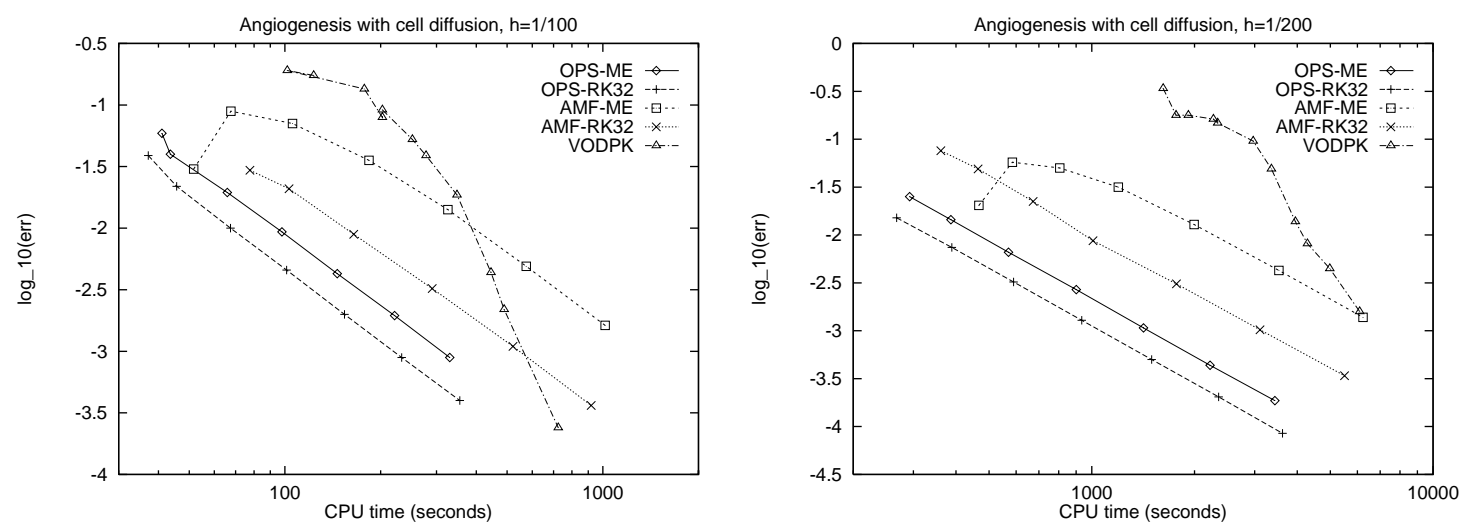

Figure 9: Results for the angiogenesis model with cell diffusion on a spatial grid with $h=1 / 100$ (left) and $h=1 / 200$ (right). AMF-RK32 failes for $T O L=10^{-3}$ in the left plot, and additional points for $T O L$ up to $10^{-8}$ are plotted for VODPK.
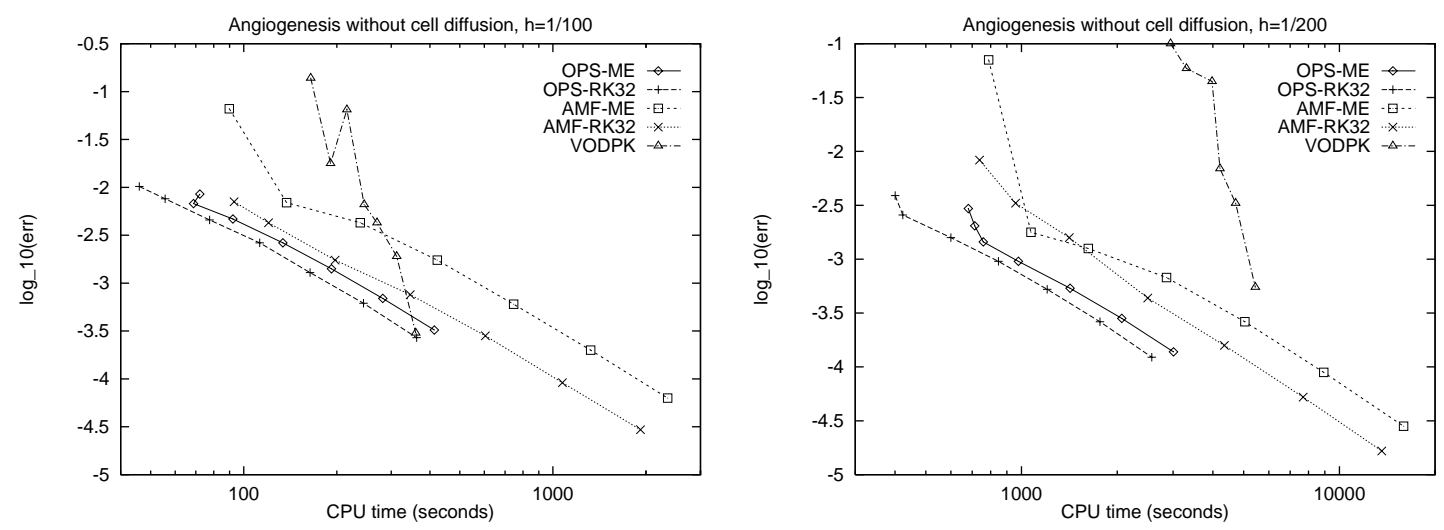

Figure 10: Results for the angiogenesis model without cell diffusion on a spatial grid with $h=1 / 100$ (left) and $h=1 / 200$ (right). VODPK failes for $T O L=10^{-3}$ in the case of $h=1 / 200$.

kind of problems under consideration.

Table 1 gives the number of performed and rejected steps of the different methods for the test case without cell diffusion on the coarse grid. The number of steps for the OPS scheme given in the table should be doubled because each counted step consists of two half steps taken in the Richardson procedure. Even with this doubling, the number of time steps taken by the OPS schemes is considerably less than those taken by the AMF methods or VODPK. This certainly is a valuable property of the OPS schemes which will pay off even more if right-hand side evaluations become more expensive. We further observe that the schemes based on RK32 require less steps than their counterparts based on ME to achieve the same accuracy. Improved stability (and maybe also positivity) properties of RK32 should be the reason for this. All these observations manifest themselves in the accuracy-CPU time plots in Figures 9 and 10.

A look at the development of the most negative component of the solution during the course of integration reveals that as soon as this value becomes too negative then there will be a rejected step. After this rejected step the integration can proceed or, especially for very low tolerances, more rejected steps follow. Possible ways out of this problem are to apply clipping to each step solution, i.e., setting all negative solution values to zero, or to employ the methods with a sufficiently high accuracy requirement. The first approach interferes with mass conservation but the effect should in general be minimal, see e.g. [23]. However, as there are only few step rejections in the tolerance 


\begin{tabular}{|c|c|c|c|c|c|}
\hline & OPS-ME & OPS-RK32 & AMF-ME & AMF-RK32 & VODPK \\
\hline TOL & steps (rej) err & steps (rej) err & steps (rej) err & steps (rej) err & steps (rej) err \\
\hline $10^{-3}$ & $60(10)-2.07$ & $35(2)-1.99$ & $175(1)-1.18$ & $148(7)-2.15$ & $506(0)-0.86$ \\
\hline $10^{-4}$ & $75(0)-2.33$ & $60(0)-2.34$ & $463(0)-2.37$ & $306(0)-2.76$ & $641(0)-1.19$ \\
\hline $10^{-5}$ & $157(0)-2.85$ & $126(0)-2.89$ & $1444(0)-3.22$ & $941(0)-3.55$ & $821(2)-2.37$ \\
\hline $10^{-6}$ & 337 ( 1) -3.49 & $278(2)-3.57$ & $4557(0)-4.20$ & $2953(0)-4.53$ & $1028(3)-3.52$ \\
\hline
\end{tabular}

Table 1: Integration statistics for the angiogenesis model without diffusion on the coarse grid $(h=1 / 100)$. The number of steps is including rejected steps but rejected steps on integration start-up are not counted. The achieved error err is given as logarithm to the base 10. The number of steps for the OPS schemes should be doubled because each counted step consists of two half steps taken in the Richardson procedure.

range considered here, no additional measures were taken.

\section{Discussion}

We have solved semi-discretizations of two taxis-reaction-diffusion models from mathematical biology by splitting methods. The two splitting approaches differ mainly in the way how the taxis discretization is treated in the time stepping process: AMF does not split the system at the ODE level but neglects the taxis part in the Jacobian approximation used in the scheme and OPS, based on Strang-splitting, separates the taxis and the reaction-diffusion parts completely. It is of special advantage for the OPS schemes that there we only split stiff from nonstiff parts of the ODE and hence the splitting error is small. The splitting error is expected to be larger if also stiff processes are split by a Strang-type scheme but in our methods this part $\left(F_{1}\right)$ is treated with the AMF approach and so we circumvent this difficulty.

Our numerical experiments also demonstrated that the choice of the underlying ERK scheme is important for the performance of the splitting methods. The RK32 scheme appears to be a suitable candidate.

The comparison of the splitting schemes with the standard integrator VODPK shows the potential of the OPS and AMF methods. This is especially true as we have not tuned our schemes for the given examples. For instance we compute and decompose the required Jacobians in our schemes for every time step even if they are constant (e.g. for the diffusion discretization) in our special cases. We have done so in order to give our numerical test results a more general meaning.

Finally, we would recommend the application of OPS-RK32 or AMF-RK32 for the simulation of taxis-reaction-diffusion models in the lower and moderate accuracy range. For the angiogenesis problem, the operator splitting scheme OPS-RK32 performs best overall.

\section{References}

[1] A. R. A. Anderson, M. A. J. Chaplain, E. L. Newman, R. J. C. Steele, and A. M. Thompson. Mathematical modelling of tumour invasion and metastasis. J. Theoret. Med., 2:129-154, 2000 .

[2] R. M. Beam and R. F. Warming. An implicit finite-difference algorithm for hyperbolic systems in conservation-law form. J. Comput. Phys., 22:87-110, 1976.

[3] C. Bolley and M. Crouzeix. Conservation de la positivité lors de la discrétisation des problèmes d'évolution paraboliques. RAIRO Anal. Numer., 12:237-245, 1978.

[4] G. D. Byrne. Pragmatic Experiments with Krylov Methods in the Stiff ODE Setting. In 'Computational Ordinary Differential Equations', J. Cash and I. Gladwell, eds., Oxford Univ. Press, Oxford, pp. 323-356, 1992. 
[5] M. A. J. Chaplain and A. M. Stuart. A model mechanism for the chemotactic response of endothelial cells to tumour angiogenesis factor. IMA J. Math. Appl. Med. Biol., 10:149-168, 1993.

[6] E. G. D'yakonov. Difference systems of second order accuracy with a divided operator for parabolic equations without mixed derivatives. USSR Comput. Math. Math. Phys., 4(5):206$216,1964$.

[7] A. Gerisch, D. F. Griffiths, R. Weiner, and M. A. J. Chaplain. A positive splitting method for mixed hyperbolic-parabolic systems. Numer. Methods Partial Differential Eq., in press, 2000.

[8] A. Gerisch and H. Podhaisky. Splitting methods for the simulation of tumor angiogenesis models. In Proceedings of the 16th IMACS World Congress 2000, Lausanne, Switzerland, M. Deville and R. Owens, eds., published on CD-ROM (ISBN 3-9522075-1-9), 2000.

[9] A. Gerisch and R. Weiner. On the positivity of low order explicit Runge-Kutta schemes applied in splitting methods. Reports on Numerical Mathematics 35, Martin-Luther-University HalleWittenberg, 1999.

[10] E. Hairer and G. Wanner. Solving ordinary differential equations II - stiff and differentialalgebraic problems. Springer Series in Computational Mathematics 14, 2nd edition, SpringerVerlag, Berlin, 1996.

[11] Zoltán Horváth. Positivity of Runge-Kutta and diagonally split Runge-Kutta methods. Appl. Numer. Math., 28:309-326, 1998.

[12] P. J. van der Houwen and B. P. Sommeijer. Approximate factorization for time-dependent partial differential equations. CWI Report MAS-R9915, Amsterdam, 1999.

[13] W. Hundsdorfer, B. Koren, M. van Loon, and J. G. Verwer. A positive finite-difference advection scheme. J. Comput. Phys., 117:35-46, 1995.

[14] W. Hundsdorfer. Accuracy and stability of splitting with stabilizing corrections. CWI Report MAS-R9935, Amsterdam, 1999.

[15] J. F. B. M. Kraaijevanger. Absolute monotonicity of polynomials occurring in the numerical solution of initial value problems. Numer. Math., 48:303-322, 1986.

[16] J. F. B. M. Kraaijevanger. Contractivity of Runge-Kutta methods. BIT, 31:482-528, 1991.

[17] D. Lanser and J. G. Verwer. Analysis of operator splitting for advection-diffusion-reaction problems from air pollution modelling. J. Comput. Appl. Math., 111:201-216, 1999.

[18] D. W. Peaceman and H. H. Rachford, Jr. The numerical solution of parabolic and elliptic differential equations. J. Soc. Indust. Appl. Math., 3:28-41, 1955.

[19] L.F. Shampine. Numerical solution of ordinary differential equations. Chapman \& Hall, New York, 1994.

[20] C.W. Shu and S. Osher. Efficient implementation of essential non-oscillatory shock-capturing schemes. J. Comput. Phys., 77:439-471, 1988.

[21] G. Strang. On the construction and comparison of difference schemes. SIAM J. Numer. Anal., 5:506-517, 1968.

[22] R. Tyson, L. G. Stern, and R. J. LeVeque. Fractional step methods applied to a chemotaxis model. J. Math. Biol., 1999, in press.

[23] J. G. Verwer, E. J. Spee, J. G. Blom, and W. Hundsdorfer. A second-order Rosenbrock method applied to photochemical dispersion problems. SIAM J. Sci. Comput., 20(4):1456-1480, 1999. 